\title{
Analysis Determination of Genetic Algorithm Crossover Point Problem in Travelling Salesman Problem
}

\author{
Khairuddin Nasution ${ }^{1}$, Tasliyah Haramaini ${ }^{1}$, Oris Krianto Sulaiman ${ }^{1}$, Mhd. Zulfansyuri Siambaton ${ }^{1}$, \\ Abdurrozzaq Hasibuan', Mhd. Asaad ${ }^{2}$ \\ ${ }^{1}$ Faculty of Engineering, Universitas Islam Sumatera Utara Medan, Indonesia \\ ${ }^{2}$ Faculty of Agriculture, Universitas Islam sumatera Utara Medan, Indonesia \\ *Corresponding authorE-mail: khairudin_nst@ft.uisu.ac.id
}

\begin{abstract}
Travelling Salesman Problem (TSP) is an optimization problem that can be applied to a variety of activities. Highlights of the TSP problem is how the salesman can manage the journey so that the distance by which this is the optimum route is the best minimum distance. This study was formulated with the effect of determining the crossover point, especially probabilities of crossover in the search for the optimal route to the TSP. One goal of this research is to find the value of a good crossover probability in achieving optimal route. Crossover method used in this study is a partially mapped crossover (PMX). Selection is used Roulette wheel selection. From the tests carried out by using $20 \%, 40 \%, 60 \%, 80 \%$ and $99 \%$ can be found that a good crossover probability is $99 \%$, with the determination of mutations worth of $0.05 \%$. The number of generation is the generation 10000 . Optimal route obtained from the data bays 29. tsp is 10463 .
\end{abstract}

Keywords: TSP, optimal service, PMX, selection, mutation, Roulette wheel, bays29.tsp

\section{Introduction}

Travelling Salesman Problem (TSP) is an optimization problem that can be applied to a variety of activities such as routing. TSP optimization problem is famous and has become the standard to try computational algorithm. The subject matter of the TSP is a salesman must visit a number of cities that are known distance from one another.[1][2]

All the cities to be visited by a salesman and the city may only be visited exactly once. The problem is how the salesman can manage the journey so that the distance by which this is the optimum route is the best minimum distance.[2]

Many methods can be used to solve TSP namely Hill Climbing Method, Ant Colony System and Dynamic Programming, Simulated Annealing, Tabu Search. Another method that can be used to solve TSP is a genetic algorithm. Genetic algorithm is an algorithm that mimics the workings of genetic processes in living organisms, where there is a process of selection, recombination and mutation to get the best chromosome in a generation. [3]

One aspect which has an important role in using genetic algorithm method is the determination of recombination involving crossover point and mutation. Usually in a Genetic Algorithm, the individual is represented by a number of bits of string and recombination implemented through crossover operator that operates on pairs of individuals (parents) to produce a new string (kids) with a cross segments of the old folks. Traditionally, the number of crossover points (which determines how many segments are interchangeable) has been determined at one point or two points. [4][5]

Based on the description above, the objectives of this research are: a. To prove that determining the crossover point using Partially Mapped Crossover (PMX) can be used to solve the traveling salesman problem.

b. To get the best fitness is solved by using Partially Mapped Crossover on the traveling salesman problem.

c. To find the influence of crossover probability value in getting the optimal distance to the completion of the traveling salesman problem

\section{Literature Review}

\section{A. Genetic algorithms}

Genetic algorithms first introduced by John Holland in his book entitled "Adaptation in natural and artificial systems", and by De Jong in his book "Adaptation of the behavior of a class of genetic adaptive systems", both published in 1975, which is the basis of genetic algorithm. [5]

The genetic algorithm is a search technique that is based on the mechanism of natural selection and genetics. The genetic algorithm is different from conventional search techniques, where the genetic algorithm begins by setting the initial conditions of random solutions called population. Each individual in the population called chromosomes, which represents a solution to the problem. Chromosome is a string of symbols, which is generally a string of binary bits. Chromosome evolved through continuous iteration, called generations. In every generation, the chromosomes are evaluated by an evaluation function. [6]. To produce the next generation, a new chromosome is called the offspring, was formed through the union of two chromosomes from the initial generation 
using crossbreeding operator (crossover) or modify chromosomes using mutation carriers (mutation). A new generation is formed through the selection process some of the parent (parents) and children (offspring), according to the fitness value, and by eliminating other chromosomes in order of population size remains constant. Chromosome suite has the highest probability to be selected. After several generations, the algorithm produces the best chromosomes are expected to represent an optimal or suboptimal solutions to the problem. When $\mathrm{P}(\mathrm{t})$ and $\mathrm{C}(\mathrm{t})$ respectively are parents and offspring in a generation $t$, the general structure of the genetic algorithm can be described as follows:

Procedure: Genetic Algorithm

Begin

evaluate $P(t)$

$$
\begin{aligned}
& t \leftarrow 0 ; \\
& \text { initialize } P(t) \text {; }
\end{aligned}
$$

while (not termination condition) do

recombine $P(t)$ to yield $C(t)$;

evaluate $C(t)$;

select $P(t+)$ from $P(t)$ and $C(t)$;

$t \leftarrow t+1$;

end

end

\section{B. Crossover}

If the parent has been selected, the birth can occur. For each gene in the chromosome of new objects resulting from selection either allele of the mother or the father. The process of combining genes can be done in several ways. The simplest method is called with a single point crossover [5] [6]. This method can be demonstrated clearly using genes diencoding in binary, even so will be translated into any form of representation gene [5].

Crossover (cross-breeding) aims to increase the diversity in the population with a string of inter-crossing string obtained from previously.

\section{Partial Mapped Crossover (PMX)}

PMX created by Goldberg and Lingle. PMX is a modification of the formulation of the two-point crossovers. The important thing about PMX is crossing two points plus some additional procedures. Select a position to specify a substring at random

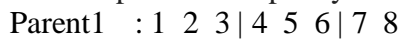

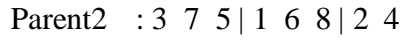

Result:

Offspring1:: 4 \begin{tabular}{lll|lll|ll} 
& 2 & 3 & 1 & 6 & 8 & 7 & 5
\end{tabular}

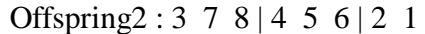

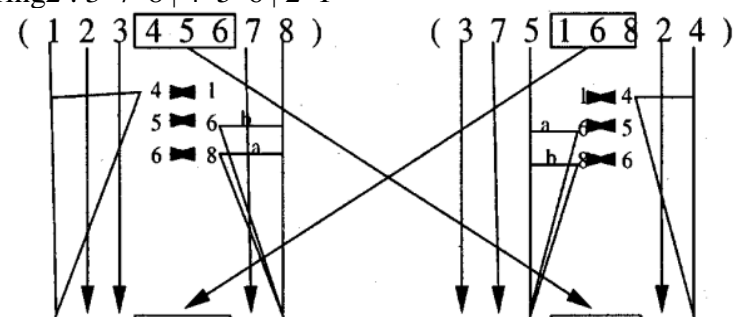

off1: ( 423316875 ) off 2 : ( 378445621 )

Fig 1: Mapping PMX[6]

D. Genetic parameters

The operation of the genetic algorithm takes four parameters, namely:

a. Probability Crosses (Crossover Probability)

b. Probability of Mutation (Mutation Probability)

c. Number of Individuals

d. Total Population

\section{Methodology}

The data used were taken from TSPLIB (TSPLIB, http://www.iwr.uniheidelberg.de/iwr/comopt/software/TSPLIB95/
). Data to be tested are: bays29.tsp. The data consists of coordinates between cities. To calculate the distance between cities Euclidean formula used is:

$d_{12}=\sqrt{\left(x_{1}-x_{2}\right)^{2}+\left(y_{1}-y_{2}\right)^{2}}$

Broadly speaking, the flow chart of this research will be carried out sequentially and systematically. All test results will be processed and conclusions are derived in the form of answers to the research objectives. Flow diagram of the study can be seen in figure 1

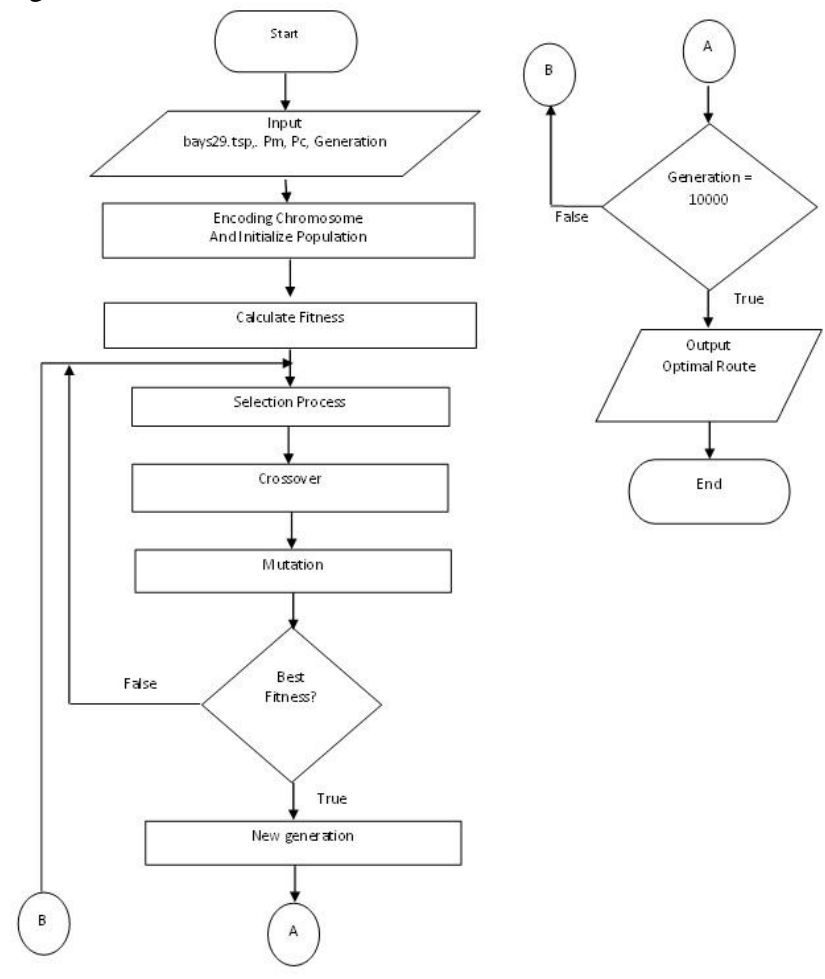

Fig 2: Flowchart Research

The stages were carried out consisting of: define the individual, defines fitness value, determine the initial population generation process, determine the selection and determine the crossover and mutation of genes. Where the stage will be carried out, namely:

a. Defining the individual, where the individual states that one solution (settlement) is possible on the issues raised.

b. Define the value of fitness, which is a measure of whether or not an individual or whether or not a solution is obtained.

c. Determine the initial population generation process. This is typically done using the random generation of such random. IPOP $=$ round \{random (Nipop, Nbits

d. Determine the selection process will be used The selection method used is the method RodaRoullete.

e. Determine the process of cross-breeding (crossover) and mutation of genes to be used.

Crossover used is Partially Mapped Crossover (PMX).

\section{Results and Discussion}

\section{Testing for Crossover Probability 20\%}

From testing to $\mathbf{p c}=\mathbf{0 . 2}$ obtained the result of the optimal route of 11008 on the generation to 9445 . Chromosome $=\begin{array}{lllll}10 & 20 & 2 & 21 & 9\end{array}$ $\begin{array}{lllllllllllllllllll}12 & 6 & 5 & 26 & 3 & 1 & 4 & 15 & 18 & 22 & 17 & 14 & 11 & 0 & 7 & 25 & 19 & 23 & 16\end{array}$ 24827 13. Fitness $=9.08385464573276$ E-05. Crossover happens as much $=1968$ and the appearance of random offsring as much $=616$. 


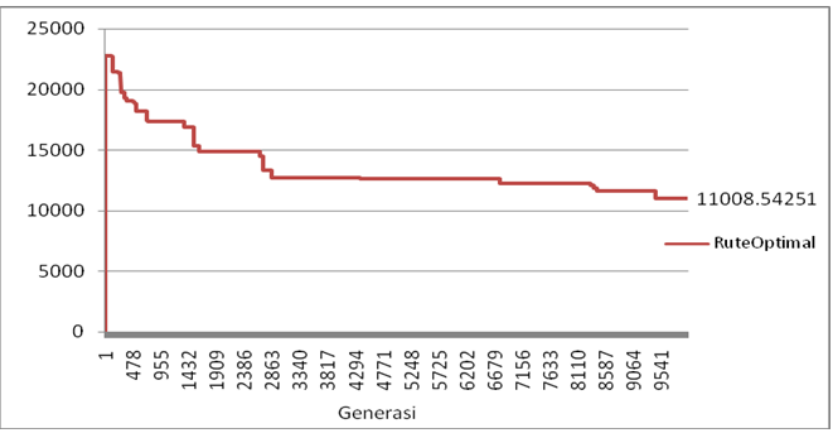

Fig 3: Route optimal on $\mathrm{pc}=0.20$

\section{Testing for Crossover Probability 40\%}

From testing to $\mathbf{p c}=\mathbf{0 . 4}$ obtained the result of the optimal route of 12488 on the generation to 9445 . Chromosome $=\begin{array}{lllll}4 & 10 & 20 & 2 & 21\end{array}$ $\begin{array}{lllllllllllllllllll}5 & 6 & 9 & 26 & 3 & 1 & 12 & 24 & 13 & 19 & 16 & 27 & 23 & 8 & 25 & 7 & 11 & 0 & 18\end{array}$ 142217 15. Fitness $=8.00742124544589 \mathrm{E}-05$. Crossover happens as much $=1968$ and the appearance of random offsring as much $=616$.

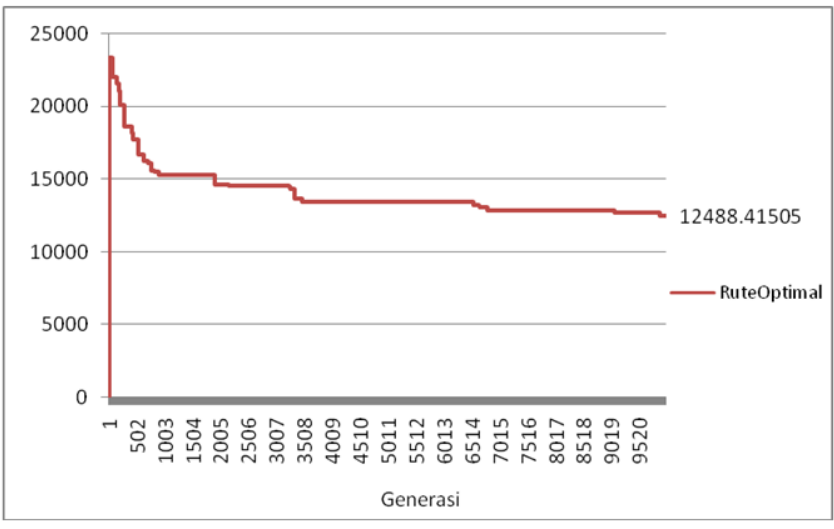

Fig 4: Route optimal on $\mathrm{pc}=0.40$

\section{Testing for Crossover Probability $60 \%$}

From testing to $\mathbf{p c}=\mathbf{0 . 6}$ obtained the result of the optimal route of 11131 on the generation to 9288 . Chromosome $=\begin{array}{lllll}17 & 22 & 11 & 0 & 7\end{array}$ $\begin{array}{lllllllllllllllllll}19 & 16 & 25 & 23 & 27 & 8 & 24 & 13 & 2 & 1 & 3 & 26 & 9 & 5 & 12 & 6 & 21 & 20 & 10\end{array}$ 41815 14. Fitness $=8.98374881702383 \mathrm{E}-05$. Crossover hap pens as much $=6095$ and the appearance of random offsring as much $=73$.

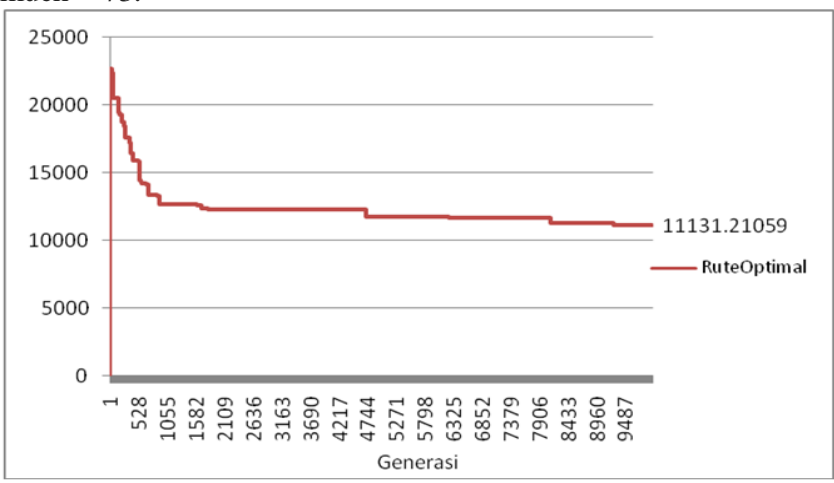

Fig 5: Route optimal on $\mathrm{pc}=0.60$

\section{Testing for Crossover Probability 80\%}

From testing to $\mathbf{p c}=\mathbf{0 . 8}$ obtained the result of the optimal route of 10548 on the generation to 9817 . Chromosome $=\begin{array}{lllll}22 & 0 & 25 & 7 & 23\end{array}$ $\begin{array}{lllllllllllllllllll}27 & 8 & 24 & 21 & 2 & 5 & 6 & 12 & 9 & 26 & 3 & 1 & 20 & 10 & 13 & 16 & 19 & 4 & 15\end{array}$ 111418 17. Fitness $=9.48039486235302 \mathrm{E}-05$. Crossover happens as much $=8071$ and the appearance of random offsring as much $=57$.

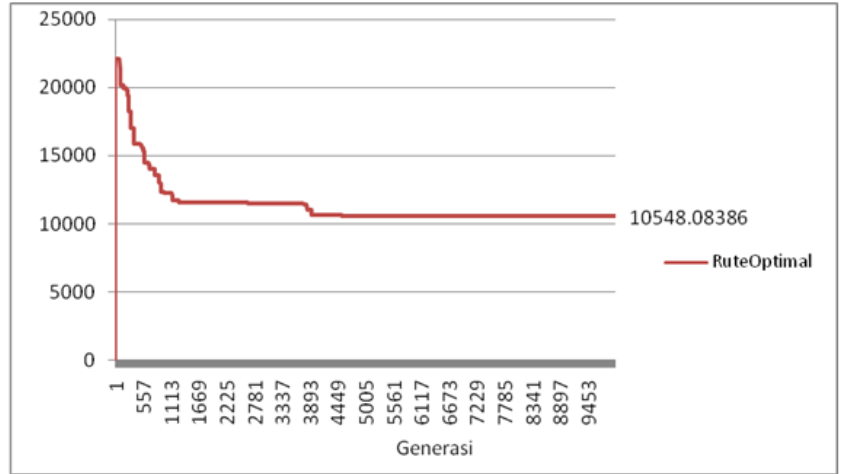

Fig 6: Route optimal on $\mathrm{pc}=0.80$

\section{Testing for Crossover Probability 99\%}

From testing to $\mathbf{p c}=\mathbf{0 . 9 9}$ obtained the result of the optimal route of 10463 on the generation to 8085. Chromosome = $19 \quad 162724$ $\begin{array}{lllllllllllllllllll}13 & 10 & 20 & 1 & 3 & 26 & 2 & 21 & 5 & 9 & 12 & 6 & 8 & 23 & 7 & 25 & 0 & 11 & 22\end{array}$ $17 \quad 14 \quad 18 \quad 15 \quad 4$. Fitness $=9.55669819112946 \mathrm{E}-05$. Crossover happens as much $=9907$ and the appearance of random offsring as much $=8$

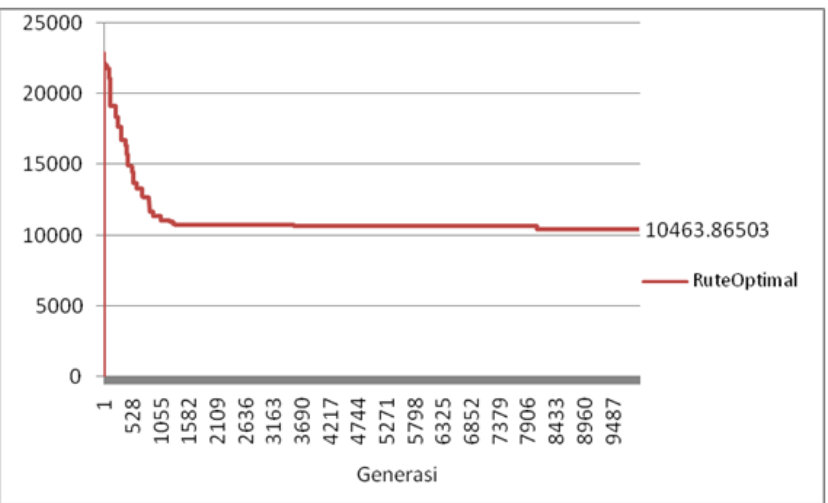

Fig 7: Route optimal on $\mathrm{pc}=0.99$

The Relationship between Crossover Probabilities with Best Fitness

From the test, can be obtained relationship between the probabilities of a crossover with the best fitness. The relationship between the probabilities of crossover with the best fitness can be illustrated as in fig. 8 .

Table 1: The relationship between the probability of crossover with the best fitness

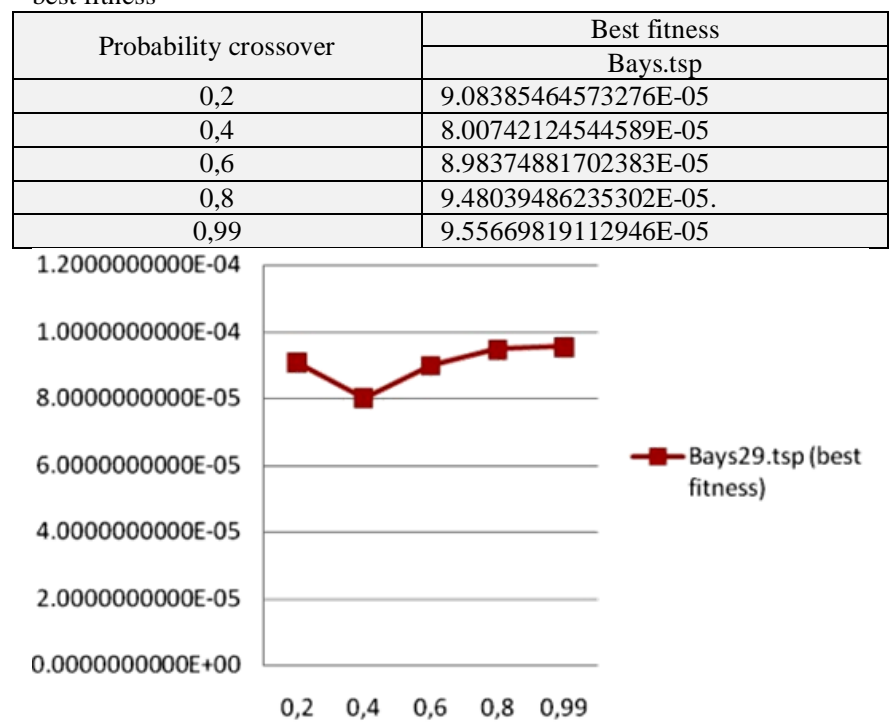

Fig 8: The relationship between the probabilities of crossover with the best fitness 


\section{The Relationship between the Probabilities of Crossover with the Optimal Route}

From some tests that have been done shows the relationship between the probabilities of crossover route optimal.

Table 2: The relationship between the probabilities of crossover with the optimal route

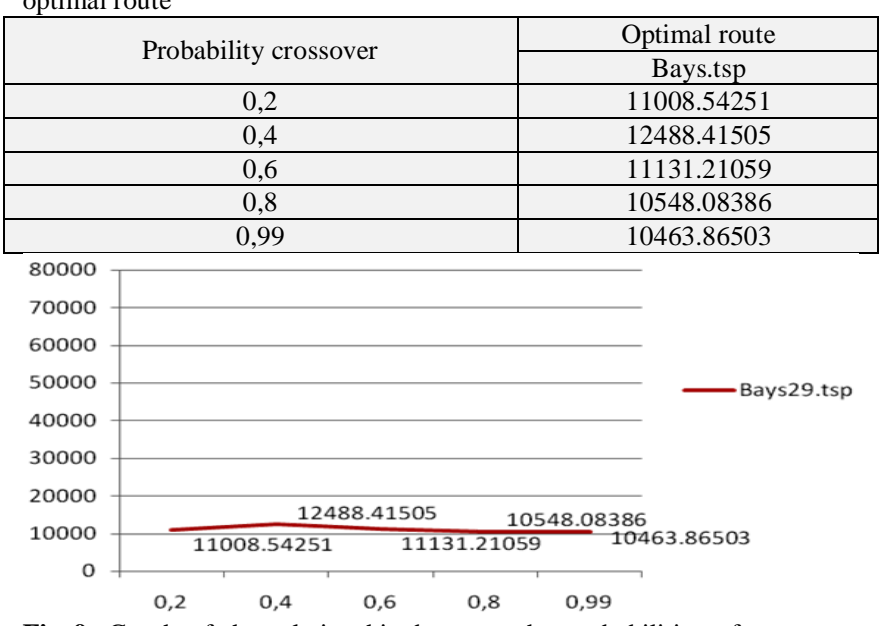

Fig 9: Graph of the relationship between the probabilities of crossover with the optimal route

From Fig 9, it can be shown that the higher the probability of crossover (pc), the more likely the optimal route is obtained.

\section{Conclusion}

This research resulted in several conclusions as follows:

a. By using Partially mapped crossover (PMX) problem Travelling Salesman Problem can be solved. In tests that have been conducted with the use of PMX crossover probability value of $99 \%$ and $5 \%$ of mutations produced the optimum distance for the generation of 10463 to 10000 for the sample data bays29.tsp.

b. In the sample data obtained bays29.tsp best fitness for 9.55669819112946E-05

c. Through the testing that has been done shows that the value of crossover probability is very influential on the optimum route search on the Travelling Salesman Problem.

\section{References}

[1] "Chapter 10 The Traveling Salesman Problem."

[2] A. R. Amin, M. Ikhsan, and L. Wibisono, "Traveling Salesman Problem," pp. 1-6, 1976.

[3] A. Fitrah and A. Zaky, "Penerapan Algoritma Genetika Pada Persoalan Pedagang Keliling ( Tsp )," pp. 3-7, 1997.

[4] Anies Hannawati, Thiang, and Eleazar, "Pencarian Rute Optimum Menggunakan Algoritma Genetika," J. Tek. Elektro, vol. 2, no. 2, pp. 78-83, 2002.

[5] L. D. Davis and M. Mitchell, "Handbook of Genetic Algorithms," VAN NOSTRAND REINHOLD, vol. 15, no. 1, pp. 4-6, 1991.

[6] M. Gen and R. Cheng, Genetic Algoritms \& Engineering Design. New York: Jhon Wiley and Sons Inc., 1997. 